\title{
Fault diagnosis of active magnetic bearings based on Gaussian GLRT detector
}

\author{
Nagel, Leon; Galeazzi, Roberto; Voigt, Andreas Jauernik; Santos, IImar
}

Published in:

3rd Conference on Control and Fault-Tolerant Systems (SysTol 2016)

Link to article, DOI:

10.1109/SYSTOL.2016.7739805

Publication date:

2016

Document Version

Early version, also known as pre-print

Link back to DTU Orbit

Citation (APA):

Nagel, L., Galeazzi, R., Voigt, A. J., \& Santos, I. (2016). Fault diagnosis of active magnetic bearings based on Gaussian GLRT detector. In 3rd Conference on Control and Fault-Tolerant Systems (SysTol 2016) (pp. 540547). IEEE. https://doi.org/10.1109/SYSTOL.2016.7739805

\section{General rights}

Copyright and moral rights for the publications made accessible in the public portal are retained by the authors and/or other copyright owners and it is a condition of accessing publications that users recognise and abide by the legal requirements associated with these rights.

- Users may download and print one copy of any publication from the public portal for the purpose of private study or research.

- You may not further distribute the material or use it for any profit-making activity or commercial gain

- You may freely distribute the URL identifying the publication in the public portal 


\title{
Fault Diagnosis of Active Magnetic Bearings based on Gaussian GLRT Detector
}

\author{
Leon Nagel ${ }^{1}$, Roberto Galeazzi ${ }^{1}$, Andreas J. Voigt ${ }^{2}$ and Ilmar F. Santos ${ }^{3}$
}

\begin{abstract}
Active magnetic bearings are progressively replacing conventional bearings in many industrial applications, particularly in the energy sector. Magnetic bearings have many advantages such as contactless support and clean operation; however their use poses also some challenges connected to their inherent open loop instability. Occurrence of faults in one or more components of an active magnetic bearing may lead to loss of control of the rotor. Timely detection and isolation of faults in an active magnetic bearing could prevent hazardous system's behaviours by enabling proper reconfiguration of the control system. A structural model of the bearing-rotor system is presented and used to perform a detectability and isolability analysis of faults in the magnetic actuator. Structural detectability and group-wise isolability is concluded for single and multiple faults in the actuator. A Gaussian generalized likelihood ratio test is proposed for detecting faults striking the electromagnet. The detector is capable to detect and isolate the occurrence of faults in e.g. the windings of bearing by tracking changes in the mean value of a Gaussian distribution. The statistical distribution of the residuals in non faulty condition is characterized by experimental data of a full-scale bearingrotor system. Verification of the detection performance is done through simulated data of a nonlinear model of the magnetic bearing calibrated against the real system.

Index Terms-Actuator fault diagnosis; Gaussian distribution; Generalized likelihood ratio test; Active magnetic bearing
\end{abstract}

\section{INTRODUCTION}

Within the last three decades the use of Active Magnetic Bearings (AMBs) as the primary bearing elements in industrial rotating machinery has seen a steady increase. Particularly in the energy sector AMBs are often favoured over conventional bearing elements in pumps, compressors and energy storage flywheels, due to their many favourable attributes. In AMB operations the shaft is levitated in a magnetic field generated by an array of electromagnetic actuators controlled through feedback of the shaft position measured by appropriate transducers [15].

An AMB is essentially a mechatronic machine element supporting a shaft without mechanical contact entailing low bearing losses and facilitating high rotational velocities. AMBs are ideal for employment in contamination sensitive application as no lubrication system is required and their

\footnotetext{
${ }^{1}$ L. Nagel and R. Galeazzi are with DTU Electrical Engineering, Technical University of Denmark, Kgs. Lyngby, 2800, Denmark LeonNagel89@gmail.com, rg@elektro.dtu.dk

${ }^{2}$ A. J. Voigt is with Lloyd Register Consulting and DTU Mechanical Engineering, Technical University of Denmark, Kgs. Lyngby, 2800, Denmark andreas. voigtelr.org

${ }^{3}$ I. F. Santos is with DTU Mechanical Engineering, Technical University of Denmark, Kgs. Lyngby, 2800, Denmark ifs@mek.dtu.dk
}

high level of controllability enables AMBs to be used for real time identification and diagnostics purposes [14].

Despite the many advantages of AMBs over conventional bearing elements, challenges do arise in their application. Operating an AMB based rotordynamic system is a complex endeavour and requires expert knowledge within a multitude of fields. Additionally, AMBs suffer from a relative low load carrying capacity compared to e.g. conventional journal bearings [14]. Furthermore, a major challenge with AMB operation is attributed to the fact that AMBs are active support elements requiring reliable performance of a number of subsystems for safe operation [15].

The electromagnetic actuator of the AMB is one such subsystem. If an actuator fails at high shaft rotational velocities, the shaft will consequently drop onto the AMB backup bearings potentially damaging the AMB system [15]. Therefore timely detection of actuator and sensor faults in AMB are of primary importance to enable any control system reconfiguration and preserve system stability.

A comprehensive study of fault scenarios for AMBs is provided in [5] where faults are categorized by type, effect and feasibility of fault tolerance. From the study of these scenarios three fault categories emerge that are not related to the rotor and are not dependent on physical redundancy for fault tolerant behaviour: actuator faults \& failures; sensor faults \& failures; software errors.

Of the three categories, actuator and sensor faults can be analysed and mitigated using structural analysis and analytical redundancy relations (ARR). Much attention have been devoted to sensor faults, see e.g. [4], [5], [12], [13], [16]. To ensure fault tolerance with respect to the AMBs both sensor and actuator faults must be mitigated.

\section{A. Contributions and novelty}

The paper offers two main contributions with respect to existing literature: first structural analyis is used to perform a detectability and isolability analysis of the faults in an AMB based rotor system focusing on the actuation part. Structural detectability and group-wise isolability is concluded for single and multiple faults in the actuator. Second, based on the statistical analysis of residuals generated through measured data a generalized likelihood ratio test (GLRT) for Gaussian random variables is presented for the detection of faults in the electromagnets. Due to the presence of correlation in the residuals the detection threshold is empirically selected based on the statistical characterization of the output of the detector. The detector is shown capable to detect and isolate 
TABLE I: Test facility specifications

\begin{tabular}{lrl}
\hline Shaft length & 860 & $\mathrm{~mm}$ \\
Shaft assembly mass & 69 & $\mathrm{~kg}$ \\
First bending mode @ & 550 & $\mathrm{~Hz}$ \\
Rotational velocity range & $0-10$ & $\mathrm{krpm}$ \\
\hline AMB Stator outer diameter & 300 & $\mathrm{~mm}$ \\
AMB rotor outer diameter & 150 & $\mathrm{~mm}$ \\
Nominal radial air gap & 0.5 & $\mathrm{~mm}$ \\
Number of poles & 8 & \\
Max. load capacity (per AMB) & 7500 & $\mathrm{~N}$ \\
Bias current range & 4 to 10 & $\mathrm{~A}$ \\
\hline
\end{tabular}

the occurrence of faults in the AMB by tracking mean value changes. Validation of the detection capabilities is performed through simulated data of a nonlinear model of the AMB calibrated against the real system.

\section{B. Experimental set-up}

The test facility utilized in this study is shown in Fig. 1. It is designed to enable component level experimental identification of rotordynamic properties of turbomachinery seals subdued to multiphase flow conditions. A detailed description can be found in [17].

Figure 1a shows a CAD rendering providing an overview of the facility and its fundamental elements. The hub components of the test facility are the two radial AMBs, the shaft and the drive unit. The radial AMBs provides contact free radial support of the shaft, which is driven by the drive unit while the shaft is axially supported by a flexible coupling between the drive unit and the shaft itself. The shaft is considered rigid in the operational range of the test facility with its first flexible mode at $f_{n, 1}=550 \mathrm{~Hz}$. The AMBs are of the eight pole heteropolar type with the four electromagnets per bearing arranged into two actuator pairs that can be controlled individually by employing e.g. a decentralized PID control scheme. The actuators are tilted $45^{\circ}$ with respects to the global reference frame so that the gravitational load of the shaft is compensated for by both actuators in each bearing. The basic specification of the test facility are summarized in Table I.

\section{MAThematical Model}

The AMB-rotor system consists of three major elements: the drive unit, the active magnetic bearings and the rotor. In the following only the latter two are analysed in order to derive a dynamical model of the system. The drive unit is implicitly considered by assuming that the rotor shaft rotates at an angular velocity $\Omega$.

\section{A. Magnetic bearing}

The central element of an AMB is the actuator that consists of an arbitrary array of electromagnets connected to power amplifiers. The electromagnets are paired and work in opposition such that forces of either sign can be generated. The actuator converts the desired force commands provided by the control system into actual forces applied to the rotor.

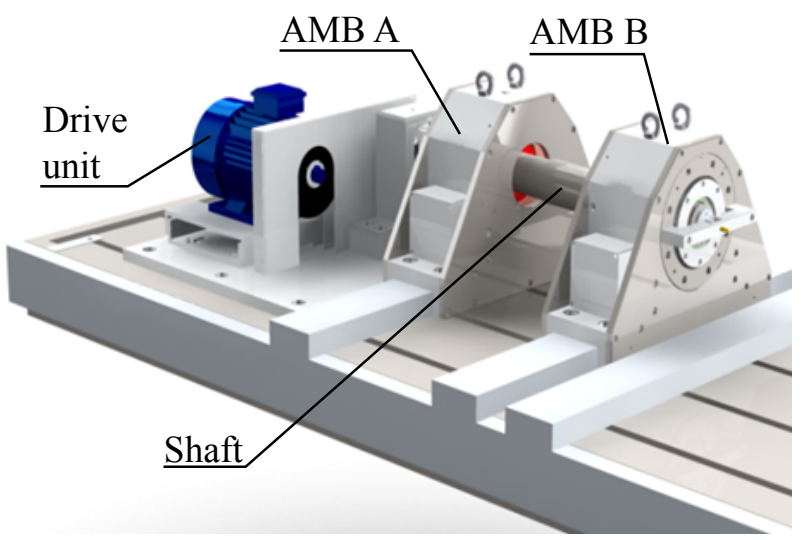

(a) $\mathrm{CAD}$ rendering providing test facility overview

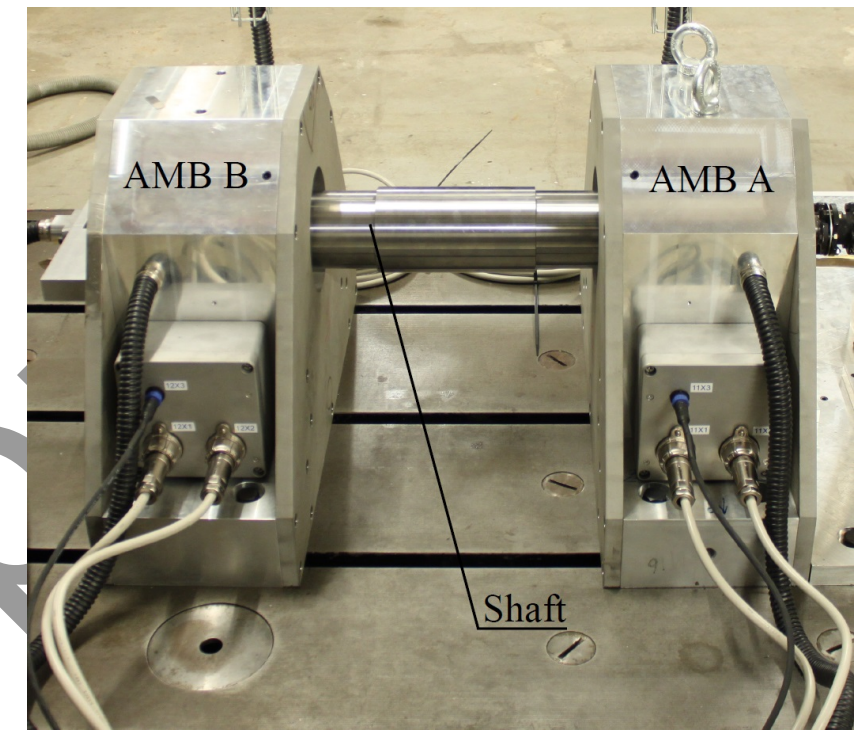

(b) Zoom view of test facility showing AMBs and shaft

Fig. 1: Illustration of the test facility

Models of the power amplifier and of the electromagnets are presented in the following to outline the fundamental working principles of an AMB.

1) Amplifier: Each opposing pair of electromagnets is connected to two power amplifiers responsible of delivering the needed current to the coil windings in response to a commanded control voltage. Several models of power amplifiers for AMBs have been discussed in literature mainly differing for the presence or not of the coil current feedback [15].

In the available test-rig the power amplifiers were provided by a third party supplier and no information about the internal structure of the amplification stages is available. Therefore the amplifiers' dynamics has been identified experimentally and the first order model

$$
G_{j}(s)=\frac{i(s)}{i_{c}(s)}=\frac{\kappa_{j}}{\tau_{p_{j}} s+1} \quad j=1, \ldots, 4 .
$$

was deemed suitable within the operational range of the bearing-rotor system. $i_{c}$ is the control current (commanded by the control system) and $i$ is the current delivered by the amplifier. In (1) $\kappa_{j}$ and $\tau_{p_{j}}$ denote the gain and time constant 


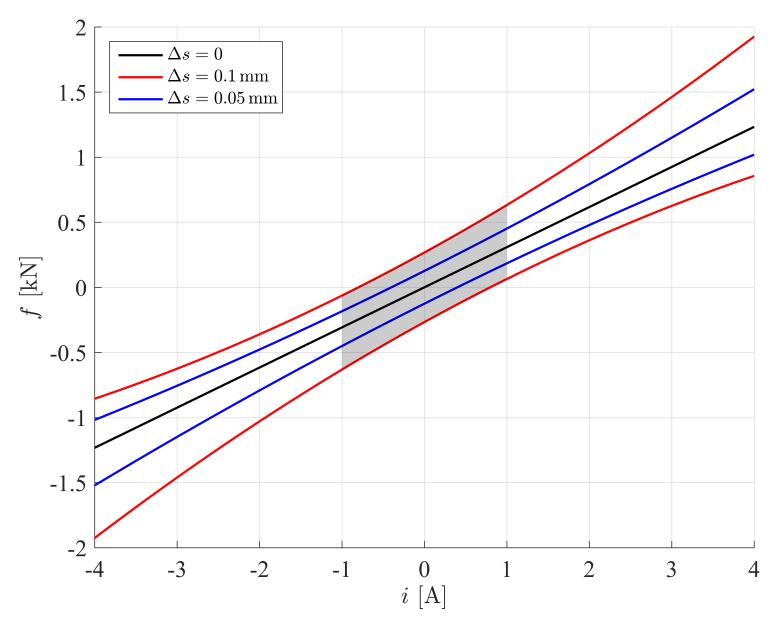

Fig. 2: Force-current characteristic of the radial bearing with $s_{0}=0.5 \mathrm{~mm}$ and bias current $i_{0}=4 \mathrm{~A}$. The shaded grey area identifies the range of the control current $i$ within which the physical test-rig was operated in stationary conditions.

identified for each amplifier.

2) Bearing electromagnets: Each magnetic bearing of the rotor dynamical system shown in Fig. 1 consists of 8 controllable electromagnets configured in differential pairs, which approximate the characteristics of a horseshoe electromagnet.

The electromagnet generates an attraction force that is proportional to the square of the coil current $i$, and inversely proportional to the square of the air gap $s$ between the bearing and the rotor, that is

$$
f(s, i)=k \frac{i^{2}}{s^{2}} \cos \alpha
$$

where $k=1 / 4 \mu_{0} n^{2} A_{a}$ is the magnetic constant with $\mu_{0}$ being the permeability of vacuum, $n$ the number of windings and $A_{a}$ the projected area of the electromagnetic poles. The term $\cos \alpha$ keeps into account that for radial magnetic bearings the forces generated by both electromagnetic poles affect the rotor at an angle $\alpha=22.5^{\circ}$.

Due to the differential pair configuration an AMB is capable of generating concomitantly attractive and repulsive forces. The common mode of operation is the so-called differential mode where one electromagnet is driven with the sum of the bias current $i_{0}$ and the control current $i$, while the opposing electromagnet is driven by the difference of the two currents. The total magnetic force is then given by

$$
f(\Delta s, i)=k\left(\frac{\left(i_{0}+i\right)^{2}}{\left(s_{0}-\Delta s\right)^{2}}-\frac{\left(i_{0}-i\right)^{2}}{\left(s_{0}+\Delta s\right)^{2}}\right) \cos \alpha
$$

where $s_{0}$ is the nominal air gap when the rotor is levitated, and $\Delta s$ is the deviation from the nominal position.

Figure 2 shows the theoretical force-current characteristic of the magnetic bearings of the test-rig, with a nominal air gap $s_{0}=0.5 \mathrm{~mm}$ and a bias current $i_{0}=4 \mathrm{~A}$. The figure shows that for up to $\pm 25 \%$ variation of the control current $i$ the magnetic force $f$ is mostly a linear function of the current also for large displacements $\Delta s$.

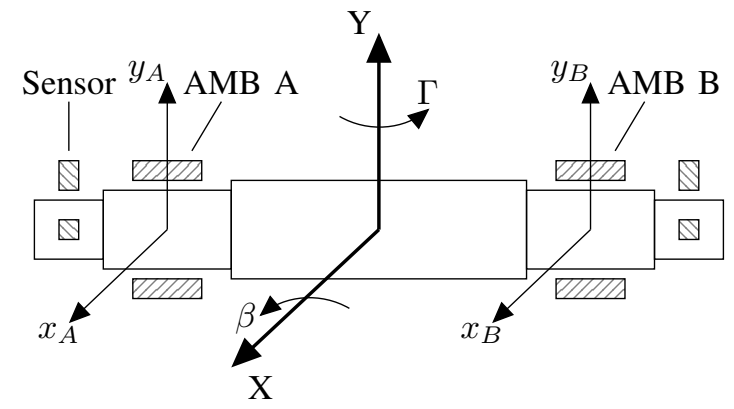

Fig. 3: Illustration of the major elements in a rotor-bearing system with the different coordinate systems used. The coordinate system of the AMBs have been rotated $45^{\circ}$ counter clockwise for illustration purposes.

\section{B. Rotor-bearing model}

The dynamical model of the rotor-bearing system is developed based on Lagrangian mechanics [6], [15]. It is developed in the actuation frame with reference to the geometric centre of the rotor. This choice results into simplified parity relations as shown in Section III. Figure 3 shows the different reference systems and relevant variables.

Let $\mathbf{q}=[x, y, \beta, \Gamma]^{\mathrm{T}}$ the vector of generalized coordinates that express the linear and angular displacements around the geometrical centre of the rotor, as shown in Fig. 3. The rotor dynamics in vectorial form is given by

$$
\mathbf{M} \ddot{\mathbf{q}}+\Omega \mathbf{G} \dot{\mathbf{q}}=\mathbf{F}\left(\mathbf{q}_{\mathrm{AMB}}, \mathbf{i}\right)+\mathbf{F}_{d}
$$

where $\mathbf{M}$ is the mass-inertia matrix, $\mathbf{G}$ is the gyroscopic matrix and $\Omega$ is the shaft rotational speed. The generalized forces and moments acting on the rotor includes two contributions: the vector of electromagnetic forces/moments $\mathbf{F}\left(\mathbf{q}_{\mathrm{AMB}}, \mathbf{i}\right)=\left[f_{x_{A}}, f_{x_{B}}, f_{y_{A}}, f_{y_{B}}\right]^{\mathrm{T}}$ function of $\mathbf{q}_{\mathrm{AMB}}=$ $\left[x_{A}, x_{B}, y_{A}, y_{B}\right]^{\mathrm{T}}$ - the rotor linear displacements within the AMBs - and $\mathbf{i}=\left[i_{x_{A}}, i_{x_{B}}, i_{y_{A}}, i_{y_{B}}\right]^{\mathrm{T}}-$ the individual control currents of each electromagnets -; the vector of external forces/moments $\mathbf{F}_{d}$ due to disturbances such as the gravitational pull $\mathbf{F}_{g}$ and mass unbalance $\mathbf{F}_{u}$. The gravitational pull is given by $\mathbf{F}_{g}=1 / \sqrt{2}[m g, m g, 0,0]^{\mathrm{T}}$ with $m$ being the total mass of the rotor shaft and $g$ the gravity constant. The mass unbalance disturbance arises when the rotor shaft rotates at an angular velocity $\Omega$ due to a misalignment between the centre of mass and the geometric centre of the rotor. It is modelled as $\mathbf{F}_{u}=\Omega^{2} m_{e} e[\sin \Omega, \cos \Omega, 0,0]^{\mathrm{T}}$ where $m_{e}$ is the added mass and $e$ is the distance between the geometric centre and the centre of mass.

Remark 1: Throughout the work it is assumed that the rotor is at rest $(\Omega=0 \mathrm{rad} / \mathrm{sec})$ hence the effect of mass unbalance is not consider further. However its magnitude can be estimated a-priori through identification procedures and hence be pre-compensated for. It is worth noting that changes to the misalignment may occur due to wear and tear of the machinery during operations, which may result into signatures in the residuals used for condition monitoring.

The dynamics of the rotor-bearing system (4) is now transformed into the bearing coordinates $\mathbf{q}_{\mathrm{AMB}}$ by means 
of the similarity transformation $\mathbf{q}_{\mathrm{AMB}}=\mathbf{T}_{r}^{b} \mathbf{q}$ where the matrix $\mathbf{T}_{r}^{b}$ is defined in (26). The model then reads

$$
\ddot{\mathbf{q}}_{\mathrm{AMB}}=-\Omega \mathbf{N} \dot{\mathbf{q}}_{\mathrm{AMB}}+\mathbf{J}\left(\mathbf{F}\left(\mathbf{q}_{\mathrm{AMB}}, \mathbf{i}\right)+\mathbf{F}_{d}\right)
$$

where $\mathbf{N} \triangleq\left(\mathbf{M} \mathbf{T}_{b}^{r}\right)^{-1} \mathbf{G} \mathbf{T}_{r}^{b}, \mathbf{J} \triangleq\left(\mathbf{M} \mathbf{T}_{r}^{b}\right)^{-1}$ and $\mathbf{T}_{r}^{b}=$ $\left(\mathbf{T}_{b}^{r}\right)^{-1}$. The constant matrices $\mathbf{N}$ and $\mathbf{J}$ have the structure

$$
\begin{aligned}
\mathbf{N} & =\left[\begin{array}{cccc}
0 & 0 & N_{13} & N_{14} \\
0 & 0 & N_{23} & N_{24} \\
N_{31} & N_{32} & 0 & 0 \\
N_{41} & N_{42} & 0 & 0
\end{array}\right] \\
\mathbf{J} & =\left[\begin{array}{cccc}
J_{11} & J_{12} & 0 & 0 \\
J_{21} & J_{22} & 0 & 0 \\
0 & 0 & J_{33} & J_{34} \\
0 & 0 & J_{43} & J_{44}
\end{array}\right], \quad J_{i, j} J_{i, j+1} \leq 0
\end{aligned}
$$

\section{Measurement model}

The position of the rotor in the radial plane is measured through four displacement sensors, which however are not collocated with the actuators. The rotor position at the AMBs' location can be then computed through the similarity transformation $\mathbf{T}_{r}^{s}$ as shown in (28). In addition to the rotor position, measurements of the control currents at the input and output of the power amplifiers are also available. The measurement vector is then given by

$$
\begin{aligned}
\mathbf{y}= & {\left[y_{1}, y_{2}, y_{3}, y_{4}, y_{5}, y_{6}, y_{7}, y_{8}, y_{9}, y_{10}, y_{11}, y_{12}\right]^{\mathrm{T}} } \\
= & {\left[x_{A}, x_{B}, y_{A}, y_{B}, i_{x_{A}}, i_{x_{B}}, i_{y_{A}}, i_{y_{B}},\right.} \\
& \left.i_{x_{A, c}}, i_{x_{B, c}}, i_{y_{A, c}}, i_{y_{B, c}}\right]^{\mathrm{T}}
\end{aligned}
$$

\section{Model verification}

The model (1), (3), (5) is central for the structural detectability and isolability of faults carried out in Section III and further for the generation of the residuals to be used by the detection scheme. Hence it is essential to verify its capability to reproduce the dynamical behaviours exhibited by the real physical system.

The model coefficients were identified using closed-loop system identification techniques. Figure 4 shows the rotor position $x_{A}$ in correspondence of the AMB A for both the simulated and real system: the model tends to overpredict the magnitude of the response; however from the close-up it is noticeable that the overall dynamic behaviour is well reproduced.

It is concluded that the model is representative of the true system and hence simulated data can be used to characterize the statistical distributions of the residuals to be monitored by the detector.

\section{Detectability AND IsOlabiLity of FAULTS IN ACTIVE Magnetic BeARINGS}

To assess the possibility of detecting and isolating faults occurring in AMB based rotor systems a structural model is set-up and utilized to determine analytical redundancy relations. Those are then scrutinized to evaluate detectability and isolability of faults specifically occurring in the major components of the AMB: the amplifier and the electromagnet. Sensor faults are not consider here; however the

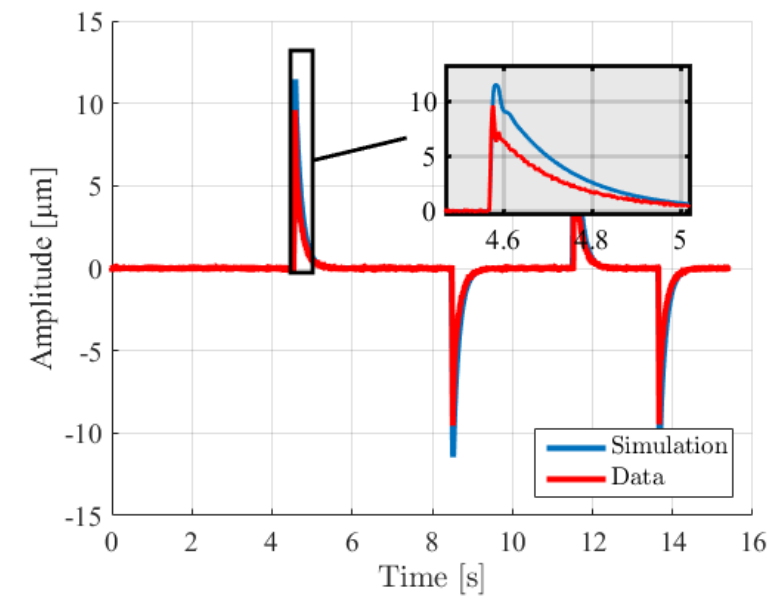

Fig. 4: Plot of the sensor output of $x_{A}$ for comparison of step response for the simulated system and the real system.

structural model can be used to extend the analysis towards this end.

\section{A. Structural analysis}

To assess the detectability and isolability properties of AMBs towards faults occurring in the amplifiers and in the electromagnets the structural analysis is performed [3, Chapter 5].

First the system variables are grouped into the set of measured variables $\mathcal{K}=\left\{i_{x_{A, c}}, i_{x_{B, c}}, i_{y_{A, c}}, i_{y_{B, c}}, i_{x_{B}}, i_{y_{A}}\right.$, $\left.i_{x_{A}}, i_{y_{B}}, x_{A}, x_{B}, y_{A}, y_{B}\right\}$ and the set of unknown variables $\mathcal{X}=\left\{\dot{x}_{A}, \dot{x}_{B}, \dot{y}_{A}, \dot{y}_{B}, \ddot{x}_{A}, \ddot{x}_{B}, \ddot{y}_{A}, \ddot{y}_{B}\right\}$. The nonlinear system (5) together with the model of the amplifiers (1) is then used to construct the incidence matrix whose analysis through the ranking algorithm [3, Chapter 5] provides the following nonlinear analytical redundancy relations

$$
\begin{aligned}
r_{1}= & \ddot{y}_{1}+\Omega N_{13} \dot{y}_{3}+\Omega N_{14} \dot{y}_{4} \\
& -J_{11} k_{x_{A}} f_{x_{A}}\left(y_{5}, y_{1}\right)-J_{12} k_{x_{B}} f_{x_{B}}\left(y_{6}, y_{2}\right) \\
r_{2}= & \ddot{y}_{2}+\Omega N_{23} \dot{y}_{3}+\Omega N_{24} \dot{y}_{4} \\
& -J_{21} k_{x_{A}} f_{x_{A}}\left(y_{5}, y_{1}\right)-J_{22} k_{x_{B}} f_{x_{B}}\left(y_{6}, y_{2}\right) \\
r_{3}= & \ddot{y}_{3}+\Omega N_{31} \dot{y}_{1}+\Omega N_{32} \dot{y}_{2} \\
& -J_{33} k_{y_{A}} f_{y_{A}}\left(y_{7}, y_{3}\right)-J_{34} k_{y_{B}} f_{y_{B}}\left(y_{8}, y_{4}\right) \\
r_{4}= & \ddot{y}_{4}+\Omega N_{41} \dot{y}_{1}+\Omega N_{42} \dot{y}_{2} \\
& -J_{43} k_{y_{A}} f_{y_{A}}\left(y_{7}, y_{3}\right)-J_{44} k_{y_{B}} f_{y_{B}}\left(y_{8}, y_{4}\right) \\
r_{5}= & \dot{y}_{5}+\tau_{p} y_{5}-\kappa y_{9} \\
r_{6}= & \dot{y}_{6}+\tau_{p} y_{6}-\kappa y_{10} \\
r_{7}= & \dot{y}_{7}+\tau_{p} y_{7}-\kappa y_{11} \\
r_{8}= & \dot{y}_{8}+\tau_{p} y_{8}-\kappa y_{12}
\end{aligned}
$$

where the constants $N_{i, j}$ and $J_{i, j}$ are the entries of the matrices $\mathbf{N}$ and $\mathbf{J}$.

\section{B. Fault detectability and isolability}

In the following analysis the concepts of structural detectability, structural isolability and strong detectability have 
to be understood in the sense of [3, Lemma 5.1, Lemma 5.2, Deinition 6.2].

Faults in the amplifiers and electromagnets are defined as parametric changes of the amplification gain and of the magnetic force gain, that is e.g.

$$
\begin{aligned}
k_{x_{A}} & =k_{0, x_{A}}\left(1-\Delta k_{x_{A}}\right) \\
\kappa & =\kappa_{0}(1-\Delta \kappa)
\end{aligned}
$$

where $\Delta k_{x_{A}}$ and $\Delta \kappa$ vary between zero (no fault) and one (complete failure). Then rewriting the faults in additive form, the fault sensitivity analysis is performed as shown in Table II, where "S" addresses the strong detectability of the fault $f_{i}$ in the residual $r_{j}$.

TABLE II: Fault sensitivity analysis

\begin{tabular}{c|cccccccc} 
& $f_{1}$ & $f_{2}$ & $f_{3}$ & $f_{4}$ & $f_{5}$ & $f_{6}$ & $f_{7}$ & $f_{8}$ \\
\hline$r_{1}$ & $\mathrm{~S}$ & $\mathrm{~S}$ & & & & & & \\
$r_{2}$ & $\mathrm{~S}$ & $\mathrm{~S}$ & & & & & & \\
$r_{3}$ & & & $\mathrm{~S}$ & $\mathrm{~S}$ & & & & \\
$r_{4}$ & & & $\mathrm{~S}$ & $\mathrm{~S}$ & & & & \\
$r_{5}$ & & & & & $\mathrm{~S}$ & & & \\
$r_{6}$ & & & & & & $\mathrm{~S}$ & & \\
$r_{7}$ & & & & & & & $\mathrm{~S}$ & $\mathrm{~S}$ \\
$r_{8}$ & & & & & & & &
\end{tabular}

Table II shows that all faults are structurally detectable, but faults in the electromagnets are not structurally isolable. However the force contributions in each of the residuals (9)(12) have opposite sign due to $J_{i, j} J_{i, j+1} \leq 0$. This entails that the fault groups $\left\{f_{1}, f_{2}\right\}$ and $\left\{f_{3}, f_{4}\right\}$ have different signatures in the residuals $\left\{r_{1}, r_{2}\right\}$ and $\left\{r_{3}, r_{4}\right\}$ when the direction of change is taken into account.

Therefore a diagnostic system capable of estimating the direction of change will achieve both detectability and isolability of all faults. The estimation of direction will further facilitate detection of group wise simultaneous faults for the two groups $\left\{f_{1}, f_{3}\right\}$ and $\left\{f_{2}, f_{4}\right\}$.

Due to the challenges in isolating faults occurring in the electromagnet this scenario is investigated further and a diagnostic system is designed based on statistical change detection theory.

\section{Detector Design And Analysis}

Statistical change detection is a consolidated discipline when the systems to be monitored are linear and the variations to be tracked are described as Gaussian stochastic process [1], [2], [11]. The problem complexity considerably increases when nonlinear systems are object of the monitoring and the processes involved are timewise correlated and/or non-Gaussian. Statistical tests can be derived but analytical methods may fall short in determining thresholds that fulfil the desired probability of detection and false alarms. Examples of condition monitoring systems tailored for such complex scenarios can be found in [7]-[9], [18].

To design a monitoring system able to detect occurrence of electromagnet faults, the statistical distribution of the residuals $\left\{r_{1}, r_{2}, r_{3}, r_{4}\right\}$ is first determined in healthy and faulty
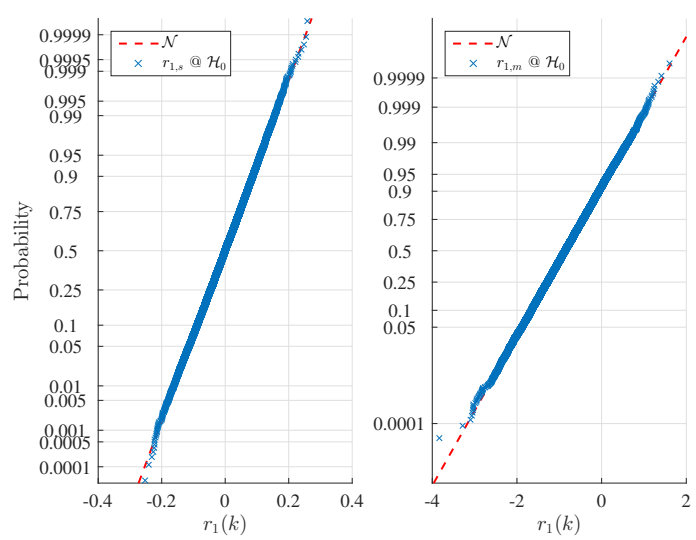

Fig. 5: Probability plot of the residual $r_{1}$ computed using simulated data (left) and measured data (right) in healthy conditions. The residual is clearly Gaussian distributed.

conditions. Once the distributions are known the statistical test can be designed. The -likelihood ratio test is one of the most applied methods because it maximizes the detection probability given the desired false alarm probability [11, Chapter 6].

Since the parameters of the statistical distribution in faulty conditions depend on the unknown magnitude of the fault they are themselves a-priori unknown. Hence the GLRT is employed because it relies on the real time computation of the maximum likelihood estimate (MLE) of the parameters.

\section{A. Residuals analysis in healthy and faulty conditions}

The statistical distributions of the residuals $\left\{r_{1}, r_{2}, r_{3}, r_{4}\right\}$ in healthy and faulty conditions are now analysed. For the healthy case simulated and measured data are utilized to generated the residuals. For the faulty case only simulated data are available.

Figure 5 shows the probability plot of the residual $r_{1}$ computed using both simulated data $\left(r_{1, s}-\right.$ left plot) and measured data $\left(r_{1, m}\right.$ - right plot). The residual is clearly Gaussian distributed. Residuals $\left\{r_{2}, r_{3}, r_{4}\right\}$ share the same statistical characteristics. This result was not anticipated due to the presence of the nonlinear magnetic force components $f_{x_{A}}$ and $f_{x_{B}}$ entering the residual equation (9), which were expected to distort the Gaussian nature of the noise affecting the measurements.

In stationary conditions the horizontal $\left\{x_{A}, x_{B}\right\}$ and vertical $\left\{y_{A}, y_{B}\right\}$ displacements of the AMBs range between $\pm 0.5 \mu \mathrm{m}$ giving rise to control current variations of up to $0.2 \mathrm{~A}$ around the bias current $i_{0}$. According to force-current characteristic shown in Fig. 2 the magnetic force generated by the electromagnet is clearly linear in this operational range. Therefore the small variations around the equilibrium position of the rotor largely attenuate the nonlinear phenomena giving rise to a Gaussian distributed residual.

Simulated data for three different magnitudes of faults in $k_{x_{A}}$ are used to characterize the distribution of the residuals $\left\{r_{1}, r_{2}, r_{3}, r_{4}\right\}$ in faulty conditions. Figure 6 shows that the type of distributions does not change upon occurrence of 


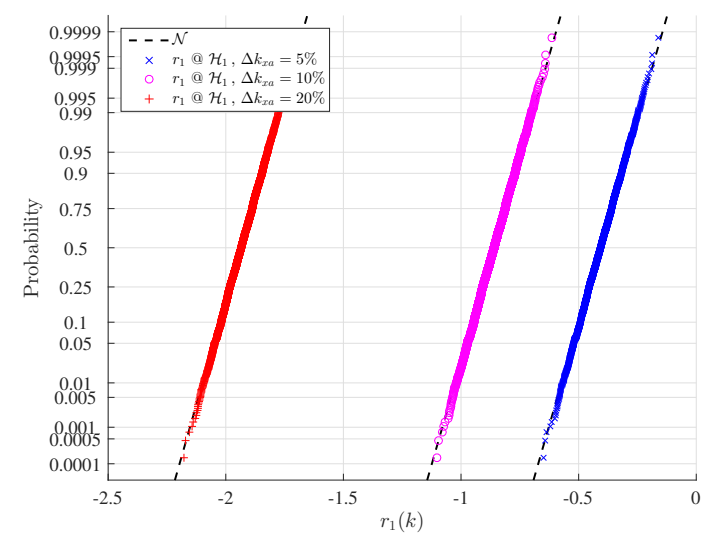

Fig. 6: Probability plot of the residual $r_{1}$ computed using simulated data for three different magnitudes of the fault $\Delta k_{x a}=\{5 \%, 10 \%, 20 \%\}$. The residual is clearly Gaussian distributed with evident change in mean value.

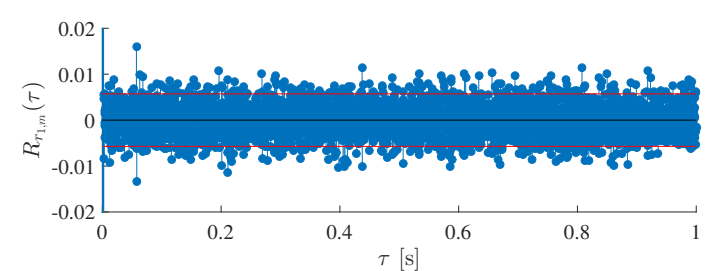

Fig. 7: Sampled autocorrelation for residual $r_{1}$ based on measured data. The residual is clearly correlated.

faults; however the mean value of the Gaussian distribution is clearly affected by the fault and it is proportional to its magnitude. Table III reports the mean value and the variance of the Gaussian distributions fitted to the residual $r_{1}$ in healthy and faulty conditions based on simulated data. It is concluded that changes in mean yalue provide a clear measure of the presence of faults in the electromagnet.

TABLE III: Gaussian distribution parameters

\begin{tabular}{ccccc}
\hline & Healthy & \multicolumn{3}{c}{ Faulty } \\
\cline { 3 - 5 } & & $\Delta k_{x_{A}}=5 \%$ & $\Delta k_{x_{A}}=10 \%$ & $\Delta k_{x_{A}}=20 \%$ \\
\hline$\mu_{\mathcal{N}}$ & 0 & -0.41 & -0.86 & -1.93 \\
$\sigma_{\mathcal{N}}$ & 0.07 & 0.07 & 0.07 & 0.07 \\
\hline
\end{tabular}

Before proceeding with the design of the statistical test the autocorrelation of the residuals is investigated, as shown in Fig. 7. The residual is clearly correlated since the sampled autocorrelation $R_{r_{1, m}}(\tau)$ lies outside the $95 \%$ confidence interval. This is not surprising as the rotor-bearing system operates in closed-loop by means of decentralized PID regulators, which through the control current introduces correlation in the system outputs.

Based on this analysis the detector must monitor unknown changes in mean value of a correlated Gaussian distributed signal. The detector is then based on the generalized likelihood ratio test for Gaussian random variables. Since no information about the correlation structure is available the design is performed assuming that the residual samples are independent and identically distributed (IID). However the assumption of being independent does not hold, therefore the detection threshold cannot be analytically computed based on asymptotic analysis of the test statistics [11, Chapter 6]. The threshold is then empirically computed based on the distribution of the detector output.

Remark 2: Another approach to overcome the correlation of the residuals is to use a whitening filter, which on the other hand may change the nature of the original distribution.

\section{B. Generalized Likelihood Ratio Test for Gaussian Dis- tributed Random Variables}

The residual $r_{i}(k), i=1, \ldots, 4$, is modelled as a Gaussian random process with mean value $\mu_{r}$ and standard deviation $\sigma_{r}$. The detection problem is to distinguish between the hypotheses [11, Chapter 6]

$$
\begin{aligned}
& \mathcal{H}_{0}: \mu_{r}=\mu_{0} \text { for } r_{i}(k), j-N+1 \leq k \leq N \\
& \mathcal{H}_{1}: \mu_{r} \neq \mu_{0} \text { for } r_{i}(k), j-N+1 \leq k \leq N
\end{aligned}
$$

A Neyman-Pearson detector decides the hypothesis $\mathcal{H}_{1}$ if the GLRT exceeds a threshold $\gamma$, that is

$$
L(\mathbf{r})=\frac{p\left(\mathbf{r}_{i} ; \hat{\mu}_{r}, \mathcal{H}_{1}\right)}{p\left(\mathbf{r}_{i} ; \mu_{0}, \mathcal{H}_{0}\right)}>\gamma
$$

where $\mathbf{r}_{i}=\left[r_{i}(j-N+1), r_{i}(j-N+2), \ldots, r_{i}(N)\right]$. Under the hypothesis that $r_{i}(k)$ are IID and considering that the residual is Gaussian distributed then (19) results in

$$
L(\mathbf{r})=\frac{\left(2 \pi \sigma_{r}^{2}\right)^{-N / 2} \exp \left(-\sum_{k=j-N+1}^{N} \frac{\left(r(k)-\hat{\mu}_{r}\right)^{2}}{2 \sigma_{r}^{2}}\right)}{\left(2 \pi \sigma_{r}^{2}\right)^{-N / 2} \exp \left(-\sum_{k=j-N+1}^{N} \frac{\left(r(k)-\mu_{0}\right)^{2}}{2 \sigma_{r}^{2}}\right)}
$$

so that the log-likelihood ratio becomes

$$
L(\mathbf{r})=\frac{N}{2 \sigma_{r}^{2}}\left(\hat{\mu}_{r}^{2}-\mu_{0}^{2}\right)
$$

where $\hat{\mu}_{r}$ is the MLE of $\mu_{r}$ under the hypothesis $\mathcal{H}_{1}[10$, Chapter 7]. Hence the detector decides $\mathcal{H}_{1}$ if

$$
g(k): \hat{\mu}_{r}^{2}>\gamma^{\prime}
$$

where $\gamma^{\prime}=\mu_{0}^{2}+2 N^{-1} \sigma_{r}^{2} \ln \gamma$.

\section{Threshold selection}

The threshold $\gamma$ is empirically determined by analysing the statistical distribution of the detector output under the hypothesis $\mathcal{H}_{0}$.

Figure 8 shows the probability plot of the test statistics $g(k)$ based on residual $r_{1}$ in non-faulty condition computed both with simulated and measured data. The behaviour of $g(k)$ is well described by the Inverse Gaussian distribution whose cumulative distribution (CDF) function is

$$
\begin{aligned}
P\left(g ; \mathcal{H}_{0}\right)= & \Phi\left(\sqrt{\frac{\lambda_{g}}{g}}\left(\frac{g}{\mu_{g}}-1\right)\right) \\
& +e^{\frac{2 \lambda_{g}}{\mu_{g}}} \Phi\left(-\sqrt{\frac{\lambda_{g}}{g}}\left(\frac{g}{\mu_{g}}+1\right)\right)
\end{aligned}
$$



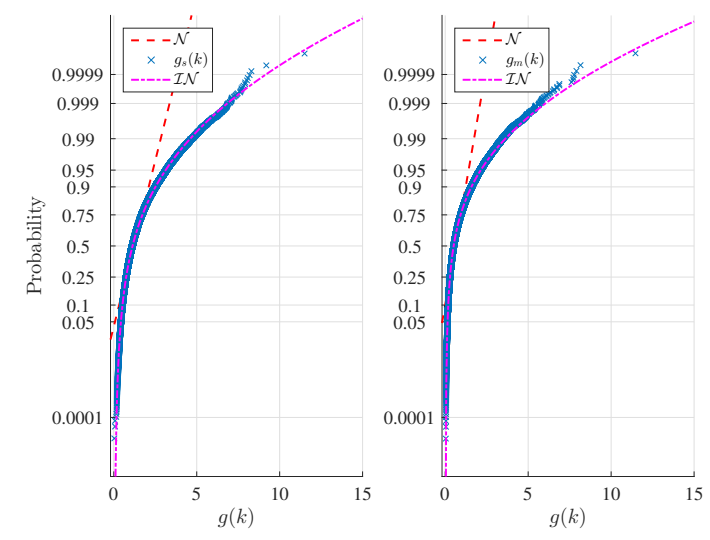

Fig. 8: Probability plot of test statistics $g(k)$ under the hypothesis $\mathcal{H}_{0}$ for simulated and measured data. In both cases the Inverse Gaussian distribution provides the best fit of the detector output.

where $\Phi(\cdot)$ is the cumulative distribution function of the Gaussian distribution, $\mu_{g}$ is the mean and $\lambda_{g}$ is the scale.

Therefore given a desired probability of false alarm $P_{F A}$ the threshold $\gamma$ is numerically computed from

$$
P_{F A}=\int_{\{g: g>\gamma\}} p\left(g ; \mathcal{H}_{0}\right) \mathrm{d} g
$$

or equivalently by

$$
1-P_{F A}=P\left(\gamma ; \mathcal{H}_{0}\right) \Rightarrow \gamma=P^{-1}\left(1-P_{F A}\right)
$$

For the given system a month interval between false alarms is deemed acceptable. With an update rate of the GLRT of $0.3 \mathrm{~ms}$ a probability of false alarm equivalent to $P_{F A} \approx 10^{-10}$ is to be achieved, which results in the threshold being set to $\gamma=24.13$.

\section{Detection Performance Verification}

The proposed method for diagnosis of faults in the electromagnets of AMBs is now tested to verify its detection capabilities. The testing is performed through simulated data since at the time of verification no faulty data were available.

Faults are injected into the simulation model presented in Section II and model responses are utilized to feed the residuals, which in turn are monitored by the Gaussian GLRT. To match as close as possible the behaviour of the true system, sampling $\left(F_{s}=3.3 \mathrm{kHz}\right)$ and quantization (16 bit resolution) of the simulated model responses have been introduced.

Verification of the detection performance through simulation study is deemed to be relevant and reliable since the statistical characterization of residuals computed on measured and simulated data performed in Section IV-A resulted in the same distribution type although with different values of parameters.

\section{A. Study case analysis}

The selected case study is a change in the electromagnetic constant $k$ that is lowered by an amount $\Delta k$. This decrease has been simulated for $k_{x_{A}}$ and $k_{x_{B}}$, thereby simulating
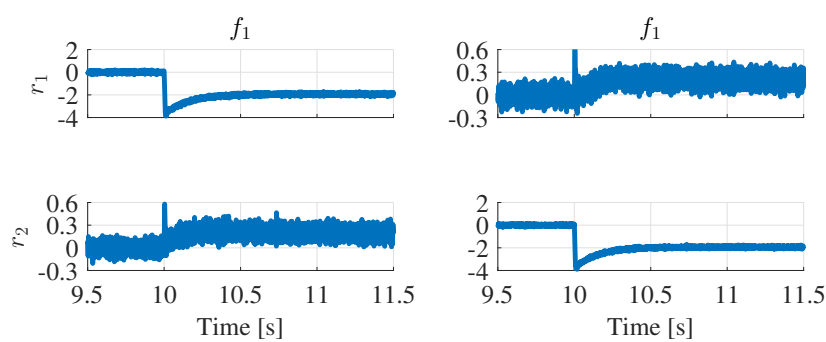

Fig. 9: Time series of the residuals $r_{1}-r_{2}$ when the AMB A is subject to a fault of magnitude $20 \%$ affecting $k_{x_{A}}$ (left plot) and when the AMB B is subject to a fault of magnitude $20 \%$ affecting $k_{x_{B}}$ (right plot).

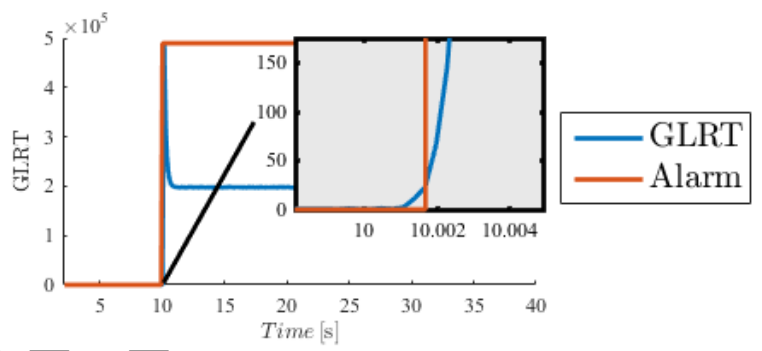

Fig. 10: Detection of fault $f_{1}$ with magnitude $\Delta k_{x_{A}}=20 \%$ by monitoring of residual $r_{1}$ through the Gaussian GLRT detector.

faults in AMB A and B. Figure 9 depicts the residuals $r_{1}$ $r_{2}$ around the fault injection time $t=10 \mathrm{~s}$ for a fault of magnitude $\Delta k=20 \%$.

It is seen that for faults $f_{1}$ and $f_{2}$, different signatures can be observed in the two residuals $r_{1}$ and $r_{2}$. This leads to isolatability of the fault by mean value estimation. This was also concluded in Section III-B.

The fault detection of three different fault amplitudes has been investigated, namely a decrease in the magnetic coefficient of $5 \%, 10 \%$ and $20 \%$. In all three cases the fault is quickly detected, and through mean value estimation is also isolated. Figure 10 shows the detection performance for a decrease in magnetic coefficient $k_{x_{A}}$ by $20 \%$ by monitoring residual $r_{1}$. The detection times $T_{D}$ for the three different fault scenarios are listed in Table IV.

TABLE IV: GLRT detection time

\begin{tabular}{ccccc}
\hline & \multicolumn{3}{c}{ Fault Magnitudes } \\
\cline { 3 - 5 } & & $\Delta k_{x_{A}}=5 \%$ & $\Delta k_{x_{A}}=10 \%$ & $\Delta k_{x_{A}}=20 \%$ \\
\hline$T_{D}$ & {$[\mathrm{~ms}]$} & 2.6 & 2 & 1.7 \\
\hline
\end{tabular}

\section{CONCLUSIONS}

The paper focused on fault diagnosis of active magnetic bearings subject to faults in the actuation. Based on a comprehensive mathematical model of the different subsystems an AMB based rotor system consists of, structural analysis was performed to highlight the general detectability and 
isolability properties of the system when subject to actuator faults. The analysis pointed out that all faults in the AMBs are structurally detectable and that full isolability can also be achieved by including an estimate of the direction of change in the diagnostic system.

The paper has further analysed the occurrence of faults in the electromagnets and a statistical characterization of the relevant residuals was performed based both on simulated and measured data from a full scale test-rig. Residuals were Gaussian distributed both in healthy and faulty cases; further occurrence of faults resulted in changes of the mean value of the distribution. Based on this analysis a Gaussian GLRT detector for changes in mean was utilized to diagnose the presence of faults in the electromagnets. Due to the correlated nature of the residuals, thresholds for detection were empirically computed based on the analysis of the GLRT output in non-faulty conditions.

The detection scheme performance was evaluated through simulated case studies by injecting faults of different magnitudes. The numerical analysis showed that fast and accurate detection of faults in the AMB's electromagnets can be achieved.

\section{APPENDIX}

\section{A. Similarity Transformations}

The actuators forces are expressed in terms of the vector $\mathbf{q}_{\mathrm{AMB}}=\left[x_{A}, x_{B}, y_{A}, y_{B}\right]^{\mathrm{T}}$ with $x_{A}$ and $x_{B}$ denoting the $x$ coordinate of bearing $\mathrm{A}$ and $\mathrm{B}$, respectively.

The coordinate transformation from $\mathbf{q}$ to $\mathbf{q}_{\mathrm{AMB}}$ is

$$
\mathbf{T}_{r}^{b} \triangleq\left[\begin{array}{llll}
1 & 0 & a & 0 \\
1 & 0 & b & 0 \\
0 & 1 & 0 & a \\
0 & 1 & 0 & b
\end{array}\right]
$$

and is derived based on trigonometric and first order approximations of sin and cos functions, with $a$ and $b$ being the longitudinal distances from the rotor centre to the bearings. Thus the relationship between the coordinates referencing the shaft geometrical centre and the actuators is $\mathbf{q}=\mathbf{T}_{b}^{r} \mathbf{q}_{\mathrm{AMB}}$.

The measured rotor position by the displacement sensors is $\mathbf{y}_{\mathrm{DS}}=\mathbf{q}_{\mathrm{DS}}+\boldsymbol{\eta}$, where $\mathbf{q}_{\mathrm{DS}}=\left[x_{A, s} x_{B, s} y_{A, s} y_{B, s}\right]^{\mathrm{T}}$ is the vector of rotor displacements in the radial plane at the sensor location and $\boldsymbol{\eta}$ is white Gaussian noise.

The coordinate transformation from $\mathbf{q}_{\mathrm{DS}}$ to $\mathbf{q}_{\mathrm{AMB}}$ is given by the combination of two similarity transformations. The output vector $q_{D S}$ can be expressed in terms of the generalized coordinates $\mathbf{q}$, that is $\mathbf{q}_{\mathrm{DS}}=\mathbf{T}_{r}^{s} \mathbf{q}$ where $\mathbf{T}_{r}^{s}$ is given by

$$
\mathbf{T}_{r}^{s} \triangleq\left[\begin{array}{llll}
1 & 0 & c & 0 \\
1 & 0 & d & 0 \\
0 & 1 & 0 & c \\
0 & 1 & 0 & d
\end{array}\right]
$$

whit $c$ and $d$ being the longitudinal distances of the sensors from the rotor centre. Then the rotor position in the actuation frame is given by

$$
\begin{aligned}
\mathbf{y}_{\mathrm{AMB}}=\mathbf{q}_{\mathrm{AMB}} & =\mathbf{T}_{r}^{b} \mathbf{q}=\mathbf{T}_{r}^{b} \mathbf{T}_{s}^{r} \mathbf{q}_{\mathrm{DS}} \\
& =\mathbf{T}_{r}^{b} \mathbf{T}_{s}^{r}\left(\mathbf{y}_{\mathrm{DS}}-\boldsymbol{\eta}\right)
\end{aligned}
$$

\section{REFERENCES}

[1] M. Basseville and I. Nikiforov, Detection of Abrupt Changes: Theory and Applications. Prentice Hall, 1993.

[2] _ , "Fault isolation for diagnosis: Nuisance rejection and multiple hypotheses testing," Annual Reviews in Control, vol. 26, no. 2, pp. 189-202, 2002.

[3] M. Blanke, M. Kinnaert, J. Lunze, and M. Staroswiecki, Diagnosis and Fault-Tolerant Control - 3rd Edition. Springer-Verlag, 2016.

[4] M. O. T. Cole, P. S. Keogh, and C. R. Burrows, "Fault-tolerant control of rotor/magnetic bearing systems using reconfigurable control with built-in fault detection," Proceedings of the Institution of Mechanical Engineers, Part C: Journal of Mechanical Engineering Science, vol. 214, no. 12, pp. 1445-1466, 2000.

[5] M. O. T. Cole, P. S. Keogh, M. N. Sahinkaya, and C. R. Burrows, "Towards fault-tolerant active control of rotormagnetic bearing systems," Control Engineering Practice, vol. 12, no. 4, pp. 491-501, 2004.

[6] B. de Kraker, Introductory Rotordynamics. Shaker Publishing, 2009.

[7] R. Galeazzi, M. Blanke, T. Falkenberg, N. K. Poulsen, N. Violaris, G. Storhaug, and M. Huss, "Parametric roll resonance monitoring using signal-based detection," Ocean Engineering, vol. 109, no. 15, pp. 355-371, 2015.

[8] R. Galeazzi, M. Blanke, and N. K. Poulsen, "Early detection of parametric roll resonance on container ships," IEEE Transactions on Control Systems Technology, vol. 21, no. 2, pp. 489-503, 2013.

[9] S. Hansen and M. Blanke, "Diagnosis of airspeed measurement faults for unmanned aerial vehicles," IEEE Transactions on Aerospace and Electronic Systems, vol. 50, no. 1, pp. 224-239, 2014.

[10] S. M. Kay, Fundamentals of statistical signal processing: estimation theory. Prentice Hall, 1993

[11] - Fundamentals of statistical signal processing: detection theory. Prentice Hall, 1998

[12] S. Kim and C. Lee, "Diagnosis of sensor faults in active magnetic bearing system equipped with built-in force transducers," IEEE/ASME Transactions on Mechatronics, vol. 4, no. 2, pp. 180-186, 1999.

[13] M. N. Sahinkaya, M. O. T. Cole, and C. R. Burrows, "Fault detection and tolerance in synchronous vibration control of rotor-magnetic bearing systems," Proceedings of the Institution of Mechanical Engineers, Part C: Journal of Mechanical Engineering Science, vol. 215, no. 12, pp. 1401-1416, 2001.

[14] G. Schweitzer, "Active magnetic bearings chances and limitations," in Proceedings of the 6th International IFToMM Conference on Rotor Dynamics, 2002.

[15] G. Schweitzer and E. H. Maslen, Eds., Magnetic Bearings - Theory, Design, and Application to Rotating Machinery. Springer-Verlag, 2009.

[16] N. Tsai, Y. King, and R. Lee, "Fault diagnosis for magnetic bearing systems," Mechanical Systems and Signal Processing, vol. 23, no. 4, pp. 1339-1351, 2009.

[17] A. J. Voigt, C. Mandrup-Poulsen, K. K. Nielsen, and I. F. Santos, "Design and calibration of a full scale active magnetic bearing based test facility for investigating rotordynamic properties of turbomachinery seals in multiphase flow," in Proceedings of the Turbomachinery Technical Conference and Exposition, 2016.

[18] A. Willeresund, M. Blanke, L. Imsland, and A. Pavlov, "Drillstring washout diagnosis using friction estimation and statistical change detection," IEEE Transactions on Control Systems Technology, vol. 23, no. 5, pp. 1886-1900, 2015. 